\title{
Population Pharmacokinetics of Sunitinib and its Active Metabolite SU012662 in Pediatric Patients with Gastrointestinal Stromal Tumors or Other Solid Tumors
}

\author{
Erjian Wang ${ }^{1} \cdot$ Steven G. DuBois ${ }^{2,3} \cdot$ Cynthia Wetmore $^{4,5,6} \cdot$ Arnauld C. Verschuur $^{7} \cdot$ Reza Khosravan $^{1}$ (1)
}

Accepted: 22 January 2021 / Published online: 14 April 2021

(c) The Author(s) 2021

\begin{abstract}
Background and Objective Population pharmacokinetic analysis explored the pharmacokinetics of sunitinib and its primary active metabolite, SU012662, in children and evaluated the sunitinib dose(s) that produce comparable plasma exposures to adults receiving the approved daily dose.

Methods Data were from 65 children with gastrointestinal stromal tumors (GIST) or solid tumors. Pharmacokinetic models of sunitinib and SU012662 were developed using a systematic multi-step approach employing nonlinear mixed-effects modeling. The effect of predefined covariates on pharmacokinetic parameters was assessed. Final models were validated using visual predictive check and statistical techniques.

Results The final dataset comprised 439 sunitinib and 417 SU012662 post-baseline plasma observations. Base models were characterized by two-compartment models with first-order absorption and lag time. Body surface area (BSA) was the only covariate that affected $(P<0.001)$ pharmacokinetic parameters for sunitinib and SU012662 and was incorporated into the final models. Bootstrap results indicated that the final models represented the final dataset adequately. Based on the final models, a sunitinib dose of $\sim 20 \mathrm{mg} / \mathrm{m}^{2} /$ day in children with GIST aged 6-17 years would be expected to lead to similar total plasma exposures of sunitinib and SU012661 as a dose of $50 \mathrm{mg}$ /day in an adult with GIST on schedule 4/2.

Conclusions In children with GIST or solid tumors receiving sunitinib, population pharmacokinetic analysis identified BSA as the only covariate that affected pharmacokinetic parameters and predicted a dose of $\sim 20 \mathrm{mg} / \mathrm{m}^{2} /$ day as achieving equivalent exposure to $50 \mathrm{mg} /$ day in adults with GIST on schedule 4/2.
\end{abstract}

Trial Registration ClinicalTrials.gov identifiers (date registered): NCT01396148 (July 2011); NCT01462695 (October 2011); NCT00387920 (October 2006).

Erjian Wang

Erjian.Wang@pfizer.com

1 Pfizer Oncology, Global Product Development, La Jolla, CA, USA

2 Department of Pediatrics, Harvard Medical School, Boston, MA, USA

3 Dana-Farber/Boston Children's Cancer and Blood Disorders Center, Boston, MA, USA

4 Center for Cancer and Blood Disorders, Phoenix Children's Hospital, Phoenix, AZ, USA

5 Department of Child Health, University of Arizona College of Medicine-Phoenix, Phoenix, AZ, USA

6 Present Address: Exelixis Clinical Development, Alameda, CA, USA

7 Department of Pediatric Hematology and Oncology, La Timone Children's Hospital, Marseille, France

\section{Key points}

Population pharmacokinetic models were used to explore the pharmacokinetics of sunitinib in children with gastrointestinal stromal tumors or other solid tumors

Based on the final model, a dose of approximately 20 $\mathrm{mg} / \mathrm{m}^{2} /$ day in children with gastrointestinal stromal tumors would be expected to lead to similar levels of sunitinib as in adults receiving the daily approved dose

This study provides new data to guide sunitinib dosing in children 


\section{Introduction}

Sunitinib is an orally available small molecule inhibitor of multiple tyrosine kinases involved in tumor proliferation and angiogenesis, including vascular endothelial growth factor receptors and platelet-derived growth factor receptors [1-4]. It is approved globally for the treatment of advanced/metastatic renal cell carcinoma (RCC) $[5,6]$ and in the US for the adjuvant treatment of adult patients at high risk of recurrent RCC following nephrectomy [5]. Sunitinib is also approved for the treatment of gastrointestinal stromal tumors (GIST) after disease progression on, or intolerance to, imatinib [5, 6]. For RCC and GIST, the approved starting dose in adults is $50 \mathrm{mg}$ taken once daily on a schedule of 4 weeks on treatment followed by 2 weeks off treatment (schedule 4/2) [5, 6]. Dose adjustments of $12.5 \mathrm{mg}$ are permitted based on individual safety and tolerability $[5,6]$.

In healthy adult volunteers or adults with solid tumors, the pharmacokinetic profile of sunitinib is well defined. The time to maximum plasma concentration of sunitinib ranges from 6 to $12 \mathrm{~h}$, and steady-state concentrations of sunitinib and its primary active metabolite SU012662 are achieved within 10-14 days [5, 6]. Sunitinib is primarily metabolized by the cytochrome P450 enzyme 3A4 (CYP3A4) to produce SU012662, which comprises $23-37 \%$ of the total exposure $[5,6]$. By day 14 , combined plasma concentrations of sunitinib and SU012662 range from 63 to $101 \mathrm{ng} / \mathrm{ml}$, which are the target concentrations predicted from preclinical data to cause anti-angiogenic and antitumor activity [1]. Following the administration of a single oral dose, the terminal half-lives of sunitinib and SU012662 are $\sim 40-60 \mathrm{~h}$ and $\sim 80-110 \mathrm{~h}$, respectively, and the apparent clearance $(\mathrm{CL} / \mathrm{F})$ of sunitinib ranges from 34 to $62 \mathrm{l} / \mathrm{h}[5,6]$.

These pharmacokinetic data in healthy adults and adults with solid tumors are supported by population pharmacokinetic models and analyses that have been used to examine covariates that might help to explain the interindividual variability in exposure of sunitinib and SU012662 and to make predictions on efficacy and safety [7-10]. Importantly, relationships between systemic exposure to the parent drug alone or the total drug (sunitinib and SU012662) and the response (efficacy and toxicities) have also been identified in adults [8-10] and in a single study in children [11]. However, to date, experience of sunitinib in pediatric patients is limited, with few pharmacokinetic studies reported [12-15]. Moreover, there have been no population pharmacokinetic analyses of sunitinib or SU012662 conducted in pediatric patients with GIST or other solid tumors. This is the first population pharmacokinetic analysis in pediatric patients that includes patients with GIST, as well as those with other solid tumors, and was conducted to address regulatory requirements and one of the key binding elements of the Sunitinib Pediatric Investigation Plan as agreed with the European Medicines Agency Pediatric Committee. Although the non-compartmental pharmacokinetic results from all three studies have been previously published in four separate articles [12-15], this population analysis pooled pharmacokinetic data from all three studies. Such population pharmacokinetic analyses further enable the characterization of the pharmacokinetics of sunitinib and SU012662 in this patient population and help to predict the dose(s) that provides comparable plasma exposures to sunitinib and SU012662 in adults receiving the approved daily dose.

The objectives of this study were to develop a population pharmacokinetic model for sunitinib and SU012662 in pediatric patients with GIST or other solid tumors; identify covariates that account for the interindividual variability in the pharmacokinetics of sunitinib and SU012662; and make predictions with respect to the pharmacokinetics of sunitinib and SU012662 in pediatric patients aged 6-11 and 12-17 years with GIST, with reference to sunitinib and SU012662 exposure in adult patients with GIST receiving the approved daily dose of $50 \mathrm{mg}$.

\section{Methods}

\subsection{Patient Population}

For this analysis, data were pooled from three studies, details of which have been published previously: ADVL0612 [12, 13]; ACNS1021 [14]; A6181196 [15]. Briefly, patients were aged between 18 months and 22 years with GIST or other solid or central nervous system (CNS) tumors, primarily high-grade glioma, ependymoma, nonspecified brain tumor, or sarcoma [12-15]. Patients received sunitinib at a starting dose of 15 or $20 \mathrm{mg} / \mathrm{m}^{2}$ on schedule 4/2, administered as an intact capsule or as capsule contents sprinkled on yogurt or applesauce [12-15]. In total, pharmacokinetic data were collected for 65 patients.

\subsection{Bioanalytical Methods}

Details of the studies relevant for the pharmacokinetic analysis, including day(s) and time point(s) of sample collections for pharmacokinetic assessment, are listed in Table 1. Sample collections for pharmacokinetic assessment were completed at prespecified visits. Plasma samples were analyzed for determination of sunitinib and SU012662 concentrations using a sensitive, specific, and validated liquid chromatography with a tandem mass spectrometry assay (BASi, West 
Table 1 Summary of included studies

\begin{tabular}{|c|c|c|c|c|c|c|}
\hline Study & $\begin{array}{l}\text { ClinicalTrials. } \\
\text { gov identifier }\end{array}$ & Phase & Tumor type & $\begin{array}{l}\text { Number of } \\
\text { evaluable } \\
\text { patients }\end{array}$ & Schedule $^{\mathrm{a}}$ and starting dose & Day(s) and time point(s) of sampling \\
\hline ADVL0612 & NCT00387920 & I & Solid tumors & 35 & $4 / 2 ; 15$ or $20 \mathrm{mg} / \mathrm{m}^{2 b}$ & $\begin{array}{l}\text { Pre-dose: days } 7,14,21 \text {, and } 28 \text { of cycle } 1 \\
\text { Post-dose: } 0,1,2,4,6,8-10 \text {, and } 24-48 \mathrm{~h} \\
\quad \text { (only for body weight }>10 \mathrm{~kg} \text { ) on day } 1 \text { of } \\
\text { cycle } 1\end{array}$ \\
\hline ACNS1021 & NCT01462695 & II & Solid tumors & 24 & $4 / 2 ; 15 \mathrm{mg} / \mathrm{m}^{2}$ & $\begin{array}{l}\text { Pre-dose (trough): days } 1,7,14 \text {, and } 28 \text { of } \\
\text { cycle } 1 \text { and days } 1 \text { and } 28 \text { of cycle } 2\end{array}$ \\
\hline A6181196 & NCT01396148 & $\mathrm{I} / \mathrm{II}$ & GIST & 6 & $4 / 2 ; 15 \mathrm{mg} / \mathrm{m}^{2}$ & $\begin{array}{l}\text { Pre-dose (trough): days } 1,15 \text {, and } 28 \text { of } \\
\text { cycles } 1-3 \text {, and optional on days } 7 \text { and } 21 \\
\text { of cycle } 1 \\
\text { Post-dose: } 2,4,6 \text {, and } 8 \mathrm{~h} \text { on day } 1 \text { of cycle } 1\end{array}$ \\
\hline
\end{tabular}

GIST gastrointestinal stromal tumor

${ }^{a}$ Schedule of 4 weeks on treatment followed by 2 weeks off treatment

${ }^{\mathrm{b}} \mathrm{An}$ amendment to the study protocol was initiated after enrolment of the first 12 patients. Starting dose in the first 12 patients was $20 \mathrm{mg} / \mathrm{m}^{2}$, reduced to $15 \mathrm{mg} / \mathrm{m}^{2}$ in the remaining patients

Lafayette, IN, USA). Further details of the bioanalytical methods are in the Supplementary Material.

\subsection{Model Development}

The pharmacokinetic modeling approaches were described in detail in the analysis plan before initiating any analysis. Regulatory guidance and quality control were taken into account. A systematic multi-step approach consisting of six stages was employed to develop a final population pharmacokinetic model: base model development, random effects model development, full model development, final model development, assessment of model adequacy (goodness of fit), and assessment of model predictive performance (validation). Analysis was conducted using nonlinear mixed-effects modeling methodology, as implemented in NONMEM (version 7.1.2, University of California at San Francisco, CA, USA) and using the first-order conditional estimation method with interaction.

Separate models were used for sunitinib and SU012662 as have been used previously in adults [7,9] and to enable direct comparison between adult and pediatric data. The base models comprised a two-compartment model with a first-order absorption rate constant $\left(k_{\mathrm{a}}\right)$ and lag time $\left(t_{\mathrm{lag}}\right)$ used to characterize the absorption process. Disposition kinetics were modeled using parameterization involving $\mathrm{CL} / \mathrm{F}$, central compartment apparent volume of distribution (Vc/F), apparent inter-compartmental clearance $(Q / F)$, and peripheral compartment apparent volume of distribution $(\mathrm{Vp} / \mathrm{F})$. Interindividual variability was modeled using multiplicative exponential random effects of the form $\theta_{i}=\theta \times$ $e^{\eta \mathrm{i}}$, where $\theta$ is the typical or central value of the parameter and $\eta i$ denoted the inter-individual random effect accounting for the ith individual's deviation from the reference value, having a mean of 0 and variance $\omega^{2}$.

Based on prior experience, groups of potential covariates were predefined for $\mathrm{CL} / \mathrm{F}, \mathrm{Vc} / \mathrm{F}$, and $k_{\mathrm{a}}$. For $\mathrm{CL} / \mathrm{F}$, these covariates were body weight or body surface area (BSA), age, sex (male or female), race (Asian or non-Asian), tumor type (GIST or other), and baseline Eastern Cooperative Oncology Group performance status (ECOG PS) of 0 or $>$ 0 , either measured directly or extrapolated from the Karnofsky performance scale. Vc/F covariates were body weight or BSA, age, sex (male or female), and tumor type (GIST or other). The $k_{\mathrm{a}}$ covariate was form (intact capsule or capsule contents sprinkled on yogurt or applesauce). Identified covariates were tested for significance using a stepwise covariate model (SCM) with the statistical criterion of $\alpha=$ 0.01 for the forward inclusion step. The full model was then subjected to a backward elimination step with the statistical criterion of $\alpha=0.001$. Covariates were eliminated one at a time, with the covariate giving the smallest increase in objective function value (OFV; the sum of squared deviations between the predictions and the observations) removed and the process repeated until all remaining covariates had a statistically significant effect on the pharmacokinetic parameter. This was the final model.

\subsection{Model Validation}

Goodness of fit of the different models was evaluated using the following criteria: change in OFV, visual inspection of diagnostic plots, precision of parameter estimates, and decreases in interindividual variability and residual variability. Diagnostic plots were examined to assess model adequacy and possible lack of fit. Plots of observed versus predicted values and observed versus individual predicted 
values were evaluated for randomness around the line of unity, and plots of conditional weighted residual versus time were evaluated for randomness around the zero line to assess whether there were any systematic deviations with respect to time that might suggest a deficiency with the structural model. Validation of the base and final models was conducted using visual predictive check (VPC) techniques comprising 1000 simulations where the median and upper and lower bounds of the $95 \%$ prediction interval (PI) for the pharmacokinetics was compared against the observed data median and confidence intervals (CIs).

\section{Results}

\subsection{Patient Characteristics and Samples}

The dataset comprised 439 sunitinib and 417 SU012662 post-baseline measurable plasma observations. In total, $27(5.8 \%)$ and $49(10.5 \%)$ post-baseline observations were below the limit of quantification (BLQ) for sunitinib and SU012662, respectively. The effect of data BLQ on modeling was not evaluated. Baseline characteristics overall and by age group (ages $2-5,6-11,12-17$, and 18-21 years) are shown in Table 2. The median (range) age was 13 (3-21) years. Overall, $33(50.8 \%)$ patients were female and 32 $(49.2 \%)$ were male.

\subsection{Sunitinib Base and Final Model}

A two-compartment model with first-order absorption including $t_{\text {lag }}$ for absorption and elimination rates was used as the base model. The correlations between the $\eta$ values for $\mathrm{CL} / \mathrm{F}$ and $\mathrm{Vc} / \mathrm{F}, \mathrm{CL} / \mathrm{F}$ and $k_{\mathrm{a}}$, and $\mathrm{Vc} / \mathrm{F}$ and $k_{\mathrm{a}}$ were weak $(0.57,-0.07$, and -0.07 , respectively); therefore, a full or partial $\omega$ block was not included in the base model.
Diagnostic plots for the base model were satisfactory, and the model appeared to be very stable, as assessed by the model eigenvalue ratio of 15.7 , which was significantly below the maximum permitted value of 1000 . Subsequently, the effect of extreme outliers on the population pharmacokinetic parameter estimates and the diagnostic plots was tested, and based on each extreme outlier observation assessment (i.e., Iconditional weighted residual [CWRES] > 6), four observations were excluded from the dataset. These were considered influential outlier observations as their exclusion led to $>15 \%$ changes in the $\omega$ of $\mathrm{CL} / \mathrm{F}, \mathrm{Vc} / \mathrm{F}$, and $k_{\mathrm{a}}$. The median values from 1000 bootstrapping analysis runs were similar to the parameter estimates of the dataset, and the 95\% CIs overlapped with those of the final dataset, suggesting that the model parameters were stable, except for $\mathrm{Vp} / \mathrm{F}$.

During the SCM analysis, the effects of different covariates on $\mathrm{CL} / \mathrm{F}$ and $\mathrm{Vc} / \mathrm{F}$ were examined. The estimated typical values for $\mathrm{CL} / \mathrm{F}$ and $\mathrm{Vc} / \mathrm{F}$ were $24 \mathrm{l} / \mathrm{h}$ and $1030 \mathrm{l}$, respectively. For $\mathrm{CL} / \mathrm{F}$, the effect of $\mathrm{BSA}$ was statistically significant $(P<0.001)$ using a power function: $\mathrm{CL} / \mathrm{F}=24$ $1 / \mathrm{h} \bullet(\mathrm{BSA} / 1.44)^{0.733}$. The effects of the other covariates of age, sex, race, tumor type, and baseline ECOG PS on CL/F were not found to be statistically significant $(P>0.001)$. For $\mathrm{Vc} / \mathrm{F}$, the effect of BSA was also statistically significant $(P<0.001)$ using a power function: $\mathrm{Vc} / \mathrm{F}=10301 \bullet$ $(\mathrm{BSA} / 1.44)^{1.46}$. The effects of the other covariates of age, sex, and tumor type on $\mathrm{Vc} / \mathrm{F}$ were not found to be statistically significant $(P>0.001)$. In a separate SCM run, BSA was replaced by baseline bodyweight. However, the final model with BSA was the model with the lower OFV and hence was selected.

Goodness-of-fit diagnostic plots and the VPC plot for plasma sunitinib concentrations from the final model are shown in Figs. 1a and 2a, respectively, and a summary of pharmacokinetic parameters in the final model and following bootstrapping is shown in Table 3 . The bootstrap results

Table 2 Patient baseline characteristics by age group

\begin{tabular}{|c|c|c|c|c|c|c|c|c|c|c|c|}
\hline \multirow[t]{2}{*}{ Age, years } & \multirow[t]{2}{*}{$n$} & \multicolumn{2}{|c|}{ Sex } & \multicolumn{2}{|l|}{ Race $^{\mathrm{a}}$} & \multicolumn{2}{|c|}{ Tumor type } & \multicolumn{2}{|c|}{$\begin{array}{l}\text { ECOG PS } \\
\text { score }^{\text {b }}\end{array}$} & \multirow[t]{2}{*}{$\begin{array}{l}\text { Median body } \\
\text { weight, kg (range) }\end{array}$} & \multirow[t]{2}{*}{ Median BSA, $\mathrm{m}^{2}$ (range) } \\
\hline & & M & $\mathrm{F}$ & Asian & Non-Asian & GIST & Other & 0 & $>0$ & & \\
\hline $2-5$ & 6 & 3 & 3 & 1 & 5 & 0 & 6 & 2 & 1 & $18.3(16.2-28.7)$ & $0.7(0.7-1.0)$ \\
\hline $6-11$ & 20 & 9 & 11 & 0 & 18 & 0 & 20 & 8 & 4 & $28.4(17.1-56.3)$ & $1.1(0.7-1.5)$ \\
\hline $12-17$ & 33 & 17 & 16 & 2 & 30 & 6 & 27 & 18 & 15 & $56(37.1-100)$ & $1.6(1.3-2.1)$ \\
\hline $18-21$ & 6 & 3 & 3 & 1 & 5 & 0 & 6 & 1 & 5 & $71.2(62.5-74.5)$ & $1.9(1.6-1.9)$ \\
\hline Total 6-17 & 53 & 26 & 27 & 2 & 48 & 6 & 47 & 26 & 19 & $49.1(17.1-100)$ & $1.4(0.7-2.1)$ \\
\hline Total 2-21 & 65 & 32 & 33 & 4 & 58 & 6 & 59 & 29 & 25 & $49.1(16.2-100)$ & $1.4(0.7-2.1)$ \\
\hline
\end{tabular}

ECOG PS Eastern Cooperative Oncology Group performance status, $F$ female, GIST gastrointestinal stromal tumor, $M$ male

${ }^{\mathrm{a}}$ Race was unknown for three patients

${ }^{\mathrm{b}}$ ECOG PS scores based on either the ECOG PS score or extrapolated from the Karnofsky Performance Status Scale, with ECOG PS scores set to 0 and 1 for Karnofsky Performance Status Scales $>90$ and $\leq 90$, respectively. The ECOG PS score could not be determined for 11 patients 


\section{a Sunitinib}

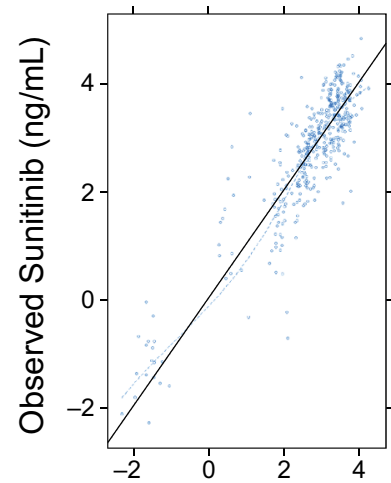

Population Predicted Sunitinib $(\mathrm{ng} / \mathrm{mL})$

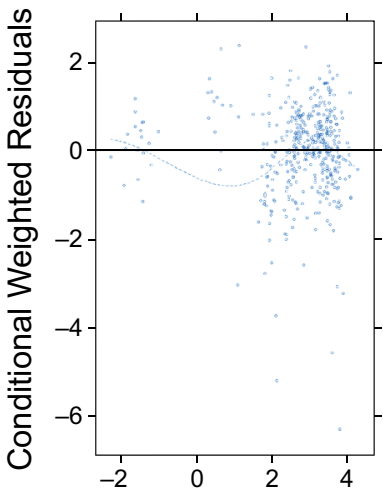

Population Predicted Sunitinib (ng/mL)

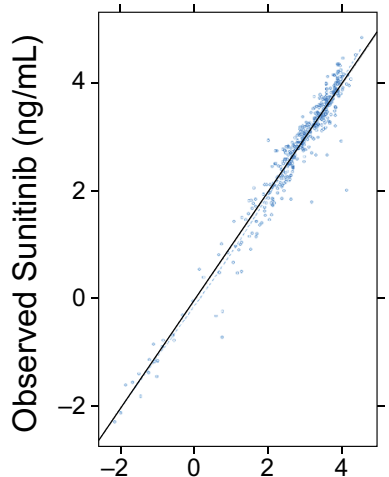

Individual Predicted Sunitinib $(\mathrm{ng} / \mathrm{mL})$

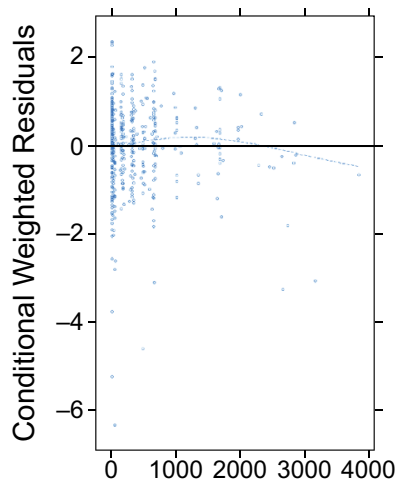

Time After First Dose

(hr)

\section{b SU012662}

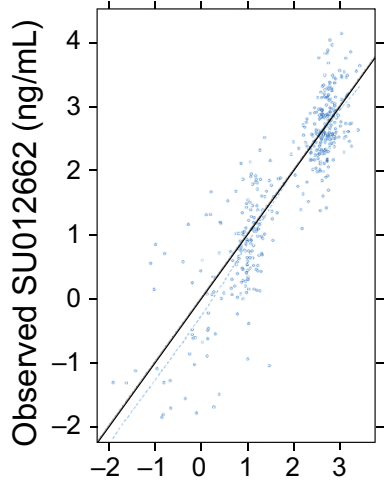

Population Predicted SU012662 (ng/mL)

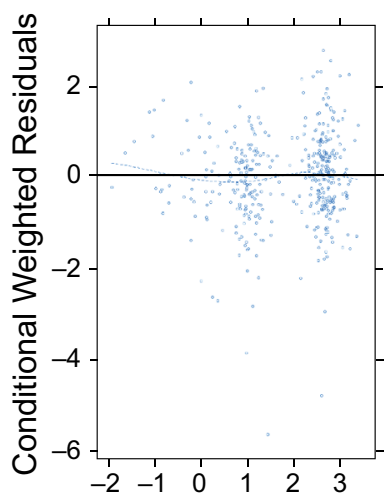

Population Predicted SU012662 (ng/mL)

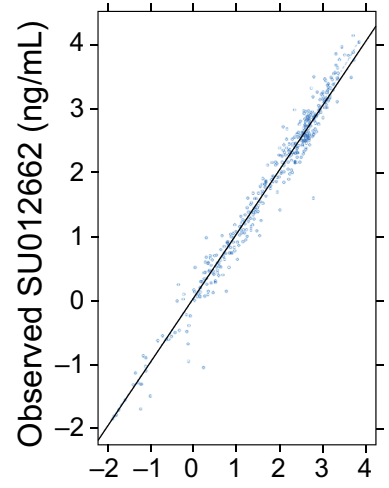

Individual Predicted SU012662 (ng/mL)

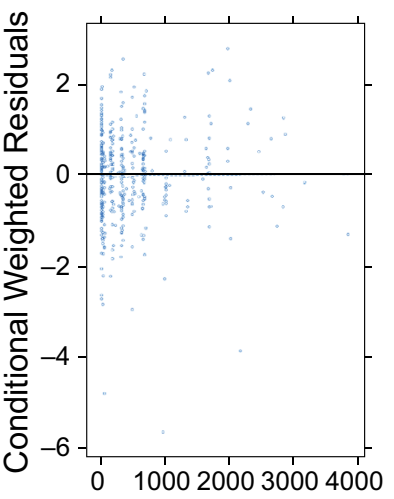

Time After First Dose

(hr)

Fig. 1 Goodness-of-fit diagnostic plots for plasma sunitinib (a) and SU012662 (b) concentrations from the final model.

were consistent with the population parameter estimates indicating that the final model was stable and that the population parameter estimates from the final model represented the final dataset adequately. Inclusion of BSA as a covariate in the final model reduced the interindividual variability of $\mathrm{CL} / \mathrm{F}$ and $\mathrm{Vc} / \mathrm{F}$ by $23.6 \%$ and $76.6 \%$, respectively.

\subsection{SU012662 Base and Final Model}

A two-compartment model with first-order absorption including $t_{\text {lag }}$ for absorption and elimination rates was used as the base model. Based on previous observations [7], a conversion of $21 \%$ sunitinib to SU012662 was assumed to bring the magnitude of the parameters to a more physiologically relevant level. The correlations between the $\eta$ values for $\mathrm{CL} / \mathrm{F}$ and $\mathrm{Vc} / \mathrm{F}, \mathrm{CL} / \mathrm{F}$ and $k_{\mathrm{a}}$, and $\mathrm{Vc} / \mathrm{F}$ and $k_{\mathrm{a}}$ were weak $(0.45,-0.13$, and -0.12 , respectively); therefore, a full or partial $\omega$ block was not included in the base model. Diagnostic plots for the base model were satisfactory, and the model appeared to be very stable, as assessed by the model eigenvalue ratio of 40.5, which was significantly below the maximum permitted value of 1000 . Subsequently, the effect of extreme outliers on the population pharmacokinetic parameter estimates and the diagnostic plots was tested, and based on each extreme outlier observation assessment (i.e., |CWRESI > 6), two observations were excluded from the dataset. These were considered influential outlier observations as their exclusion led to $>15 \%$ changes in interindividual random effects.

During the SCM analysis, the effects of different covariates on $\mathrm{CL} / \mathrm{F}$ and $\mathrm{Vc} / \mathrm{F}$ were examined. The estimated typical values for CL/F and VC/F were $11.1 \mathrm{l} / \mathrm{h}$ and $1060 \mathrm{l}$, respectively. For $\mathrm{CL} / \mathrm{F}$, the effect of $\mathrm{BSA}$ was statistically significant $(P<0.001)$ using a power function: $\mathrm{CL} / \mathrm{F}=11.1$ $1 / \mathrm{h} \bullet(\mathrm{BSA} / 1.44)^{0.87}$. The effects of the other covariates of age, sex, race, tumor type, and baseline ECOG PS on CL/F 


\section{a Sunitinib}

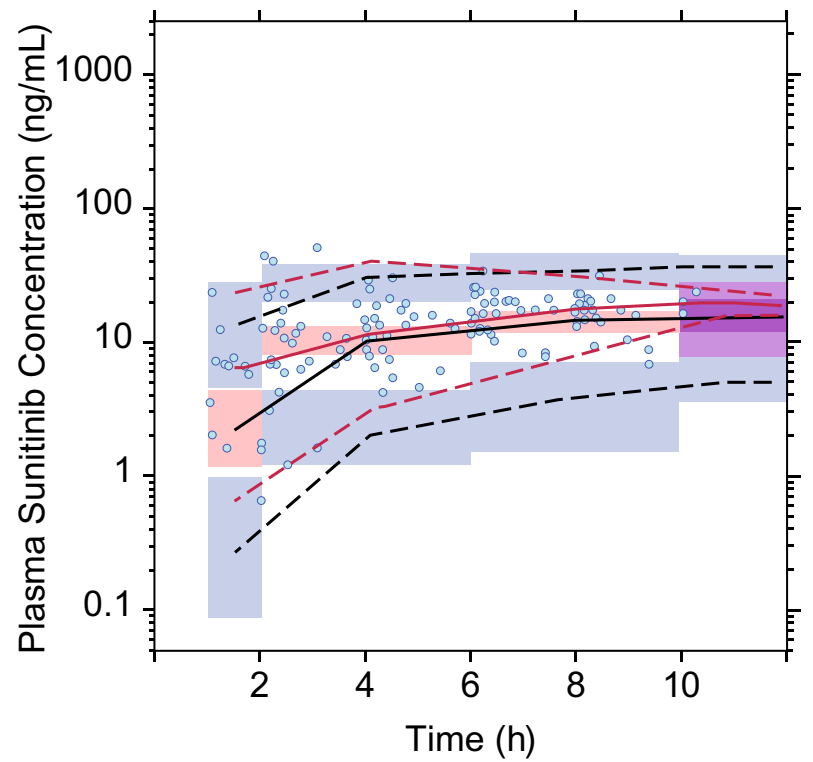

\section{c Sunitinib}

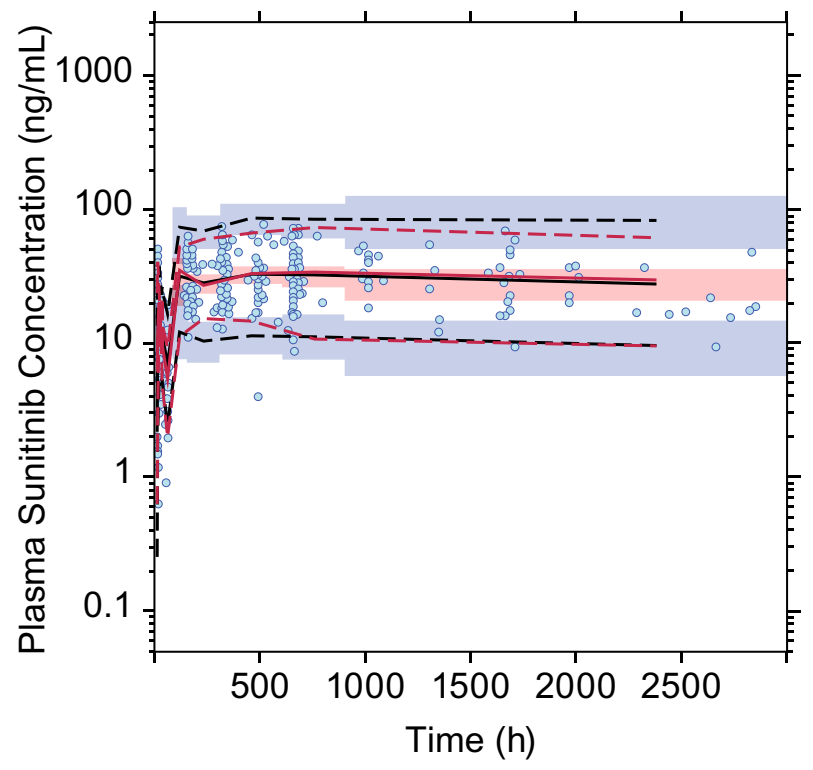

Fig. 2 Visual predictive check plots for sunitinib (a) and SU012662 (b) in the first $12 \mathrm{~h}$ post-dose and for sunitinib (c) and SU012662 (d) up to $3000 \mathrm{~h}$ post-dose. Blue circles represent the observed data, and the red lines represent the median (solid line) and 2.5th and 97.5th percentiles (dashed lines) of the observed data. The solid black line and dashed black lines represent the median and 2.5th and 97.5th per-

were not found to be statistically significant $(P>0.001)$. For $\mathrm{Vc} / \mathrm{F}$, the effect of BSA was also statistically significant $(P<0.001)$ using a power function: $\mathrm{Vc} / \mathrm{F}=10601 \bullet$ $(\mathrm{BSA} / 1.44)^{1.61}$. The effects of the other covariates of age,

\section{b SU012662}

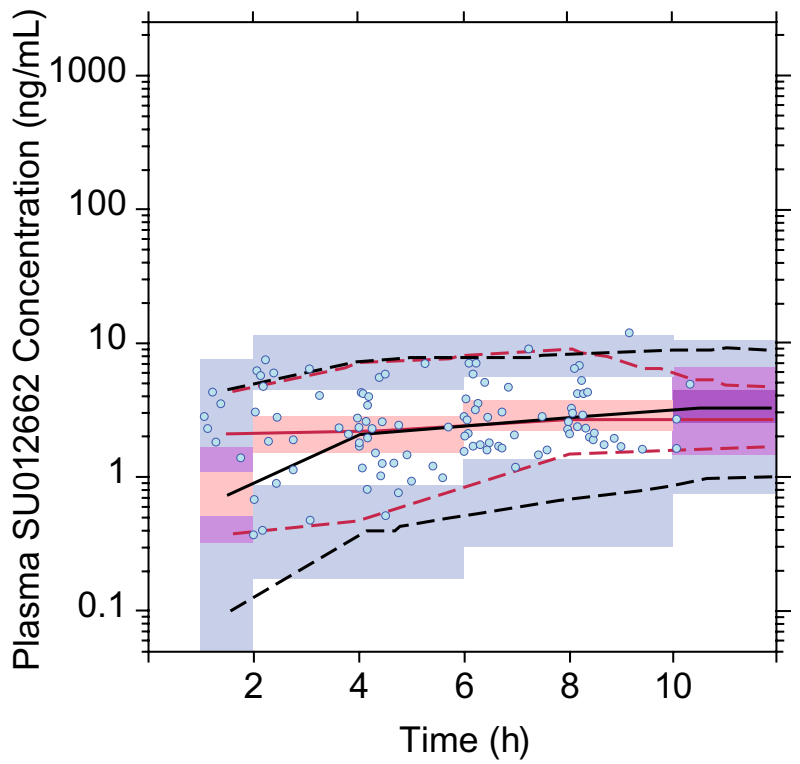

\section{d SU012662}

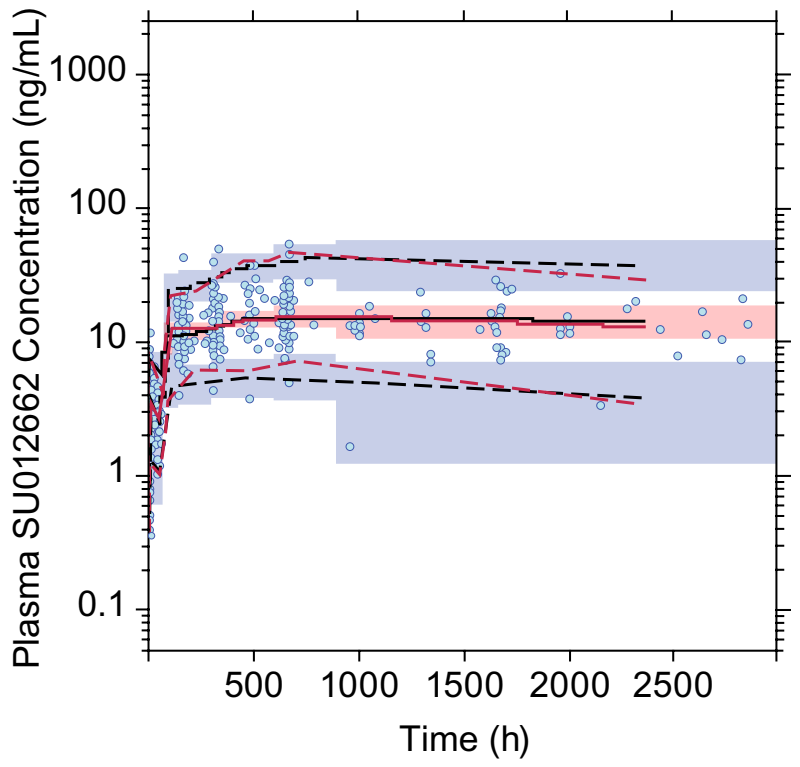

centiles of the simulated data. The $95 \%$ confidence intervals for the simulated median and each percentile are shown by the pink and blue shaded areas, respectively. Sunitinib was administered at a dose of 15 $\mathrm{mg} / \mathrm{m}^{2}$ once daily on a schedule of 4 weeks on treatment followed by 2 weeks off treatment (schedule 4/2)

sex, and tumor type on $\mathrm{Vc} / \mathrm{F}$ were not found to be statistically significant $(P>0.001)$. In a separate SCM run, BSA was replaced by baseline body weight. However, the final 
Table 3 Summary of final model pharmacokinetic parameters for sunitinib and SU012662

\begin{tabular}{|c|c|c|c|c|}
\hline \multirow[t]{2}{*}{ Parameter } & \multicolumn{2}{|l|}{ Sunitinib } & \multicolumn{2}{|l|}{ SU012662 } \\
\hline & Results, mean (RSE \%) & Bootstrap, median (95\% CI) & Results, mean (RSE \%) & Bootstrap, median $(95 \% \mathrm{CI})$ \\
\hline $\mathrm{CL} / \mathrm{F}(\theta 1), 1 / \mathrm{h}$ & $24.0(5.8)$ & $23.8(15.4-26.7)$ & $11.1(6.9)$ & $11.1(9.35-12.6)$ \\
\hline $\mathrm{Vc} / \mathrm{F}(\theta 2), 1$ & $1030(9.8)$ & $1006(845-1164)$ & $1060(14)$ & $975(543-1193)$ \\
\hline$k_{\mathrm{a}}(\theta 3), \mathrm{h}^{-1}$ & $0.37(28.3)$ & $0.35(0.23-0.62)$ & $0.28(36.7)$ & $0.26(0.15-0.37)$ \\
\hline$t_{\text {lag }}(\theta 4), \mathrm{h}$ & $0.76(3.6)$ & $0.75(0.57-0.88)$ & $0.64(26.3)$ & $0.65(0.44-0.76)$ \\
\hline $\mathrm{Vp} / \mathrm{F}(\theta 5), 1$ & $81.3(22.9)$ & $95.4(64.3-425,327)$ & $63.1(141)$ & $113(0.63-4902)$ \\
\hline$Q / F(\theta 6), 1 / \mathrm{h}$ & $0.39(79.6)$ & $0.46(0.26-11.4)$ & $6.7(319)$ & $9.75(0.09-50,575)$ \\
\hline BSA on CL/F ( $\theta 9)$ & $0.73(25.6)$ & $0.75(0.41-1.34)$ & $0.87(26)$ & $0.87(0.48-1.27)$ \\
\hline BSA on $\mathrm{Vc} / \mathrm{F}(\theta 8)$ & $1.46(19.9)$ & $1.47(0.99-1.83)$ & $1.61(20)$ & $1.82(1.23-3.52)$ \\
\hline$\omega(\mathrm{CL} / \mathrm{F}), \%$ & $33(35.4)$ & $32.7(26.5-51.2)$ & $44(19.8)$ & $42.5(30.9-55.2)$ \\
\hline$\omega(\mathrm{Vc} / \mathrm{F}), \%$ & $25.3(45.2)$ & $21.8(0.25-40.8)$ & $42(37.6)$ & $43.7(27.2-64.4)$ \\
\hline$\omega\left(k_{\mathrm{a}}\right), \%$ & $103.4(41.5)$ & $100.6(67.2-148)$ & $95.7(42.4)$ & $90(52.3-122)$ \\
\hline$\sigma(\theta 7), \%$ & $32.2(2.3)$ & $31.7(26-38)$ & $26(3.24)$ & $25.8(22-31)$ \\
\hline
\end{tabular}

$B S A$ body surface area, $C I$ confidence interval, $C L / F$ apparent clearance, $k_{a}$ first-order absorption rate constant, $t_{\text {lag }}$ lag time; $Q / F$ apparent intercompartmental clearance, $R S E$ relative standard error, $V c / F$ central compartment apparent volume of distribution, $V p / F$ peripheral compartment apparent volume of distribution, $\sigma$ residual variability, $\omega$ interindividual variability

model with BSA was the model with the lower OFV and hence was selected.

Goodness-of-fit diagnostic plots and the VPC plot for plasma SU012662 concentrations from the final model are shown in Figs. $1 \mathrm{~b}$ and $2 \mathrm{~b}$, respectively, and a summary of pharmacokinetic parameters in the final model and following bootstrapping is shown in Table 3 . The bootstrap results were consistent with the population parameter estimates indicating that the final model was stable and that the population parameter estimates from the final model represented the final dataset adequately. Inclusion of BSA as a covariate in the final model reduced interindividual variability of CL/F and $\mathrm{Vc} / \mathrm{F}$ by $32.4 \%$ and $58.0 \%$, respectively.

\subsection{Predicted Dosing of Sunitinib and SU012662 Based on the Final Population Pharmacokinetic Models}

Bootstrapping techniques were used to estimate the expected effect of BSA on values for the area under the curve (AUC) for sunitinib and SU012662 relative to a reference patient, an adult with GIST receiving a sunitinib dose of $50 \mathrm{mg} /$ day on schedule 4/2. Sunitinib and SU012662 geometric mean (minimum, maximum) AUCs in this reference adult patient with GIST were 1233 (1009, 1793) and 551 (311, 1254) $\mathrm{ng} \bullet \mathrm{h} / \mathrm{ml}$, respectively (data on file). The analysis showed that the relative AUC values for sunitinib and SU012662 for a typical patient aged 6-17 years at a sunitinib dose of $20 \mathrm{mg} / \mathrm{m}^{2}$ was predicted to be between $75 \%$ and $125 \%$ of the AUC of the reference (Fig. 3).

Using the final sunitinib and SU012662 models, the doses in pediatric patients required to achieve the steady-state 24-h adult reference sunitinib and SU012662 AUCs of 1233 and $551 \mathrm{ng} \bullet \mathrm{h} / \mathrm{ml}$, respectively, at a sunitinib dose of $50 \mathrm{mg}$ were calculated. In a typical pediatric patient aged 2-5 years (median $\mathrm{BSA}=0.69 \mathrm{~m}^{2}$ ), the predicted sunitinib dose that was expected to lead to steady-state sunitinib total plasma exposure over the dosing interval similar to that observed in adults receiving $50 \mathrm{mg}$ was $25 \mathrm{mg} / \mathrm{m}^{2}$. With respect to SU012662 total plasma exposure, the predicted sunitinib dose was $22 \mathrm{mg} /$ $\mathrm{m}^{2}$. Similarly, in patients aged 6-11 years (median BSA $=1.1 \mathrm{~m}^{2}$ ), with respect to sunitinib and SU012662 total plasma exposures, the predicted sunitinib dose was $22 \mathrm{mg} /$ $\mathrm{m}^{2}$ and $21 \mathrm{mg} / \mathrm{m}^{2}$, respectively. Finally, in patients aged $12-17$ years (median $\mathrm{BSA}=1.6 \mathrm{~m}^{2}$ ), with respect to sunitinib and SU012662 total plasma exposures, the predicted sunitinib dose was $20 \mathrm{mg} / \mathrm{m}^{2}$ and $20 \mathrm{mg} / \mathrm{m}^{2}$, respectively. Therefore, altogether, a sunitinib dose of $\sim 20 \mathrm{mg} / \mathrm{m}^{2} /$ day in pediatric patients aged 6-17 years would be expected to lead to similar total plasma exposures for sunitinib and SU012662 as compared with adult patients on $50 \mathrm{mg} /$ day on schedule $4 / 2$. Considering that the lowest dose strength for sunitinib is $12.5 \mathrm{mg}$ and that the commercially available sunitinib doses are 12.5, 25.0, 37.5, and $50.0 \mathrm{mg}$, additional analyses were performed to identify a BSA-tiered dosing strategy. The results of this analysis show that the doses in pediatric patients required to achieve the steadystate sunitinib (and SU012662) AUC approximately similar to that observed in adult patients with GIST receiving $50 \mathrm{mg} /$ day were $12.5 \mathrm{mg}, 25.0 \mathrm{mg}, 37.5 \mathrm{mg}$, and $50.0 \mathrm{mg}$ once daily on schedule $4 / 2$ for those with BSA of $\leq 0.7$ $\mathrm{m}^{2}, 0.8-1.5 \mathrm{~m}^{2}, 1.6-2.4 \mathrm{~m}^{2}$, and $\geq 2.5 \mathrm{~m}^{2}$, respectively. 


\section{a Sunitinib}

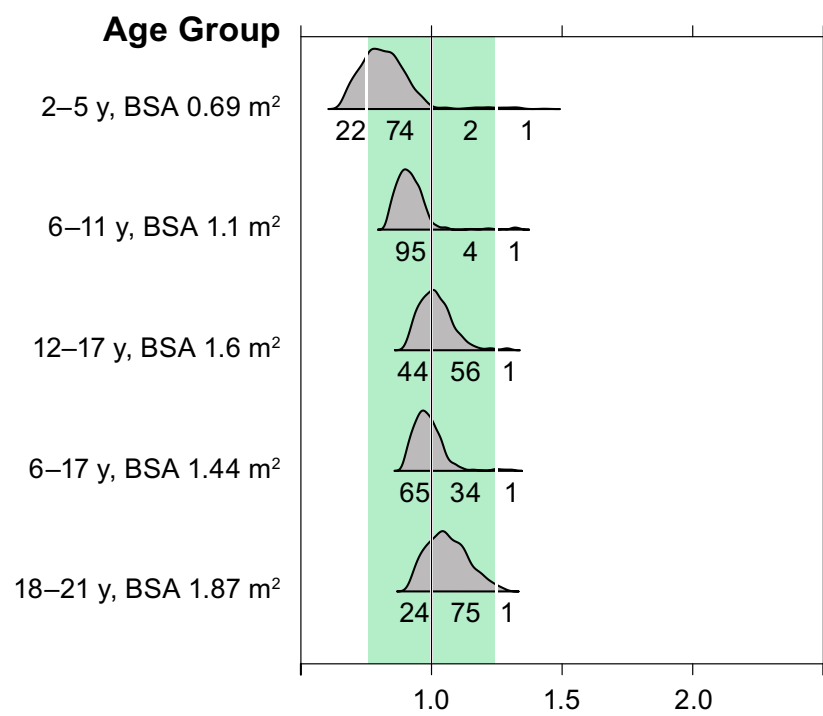

Ratio of Predicted AUC Related to the Value in Adult Reference

\section{b SU012662}

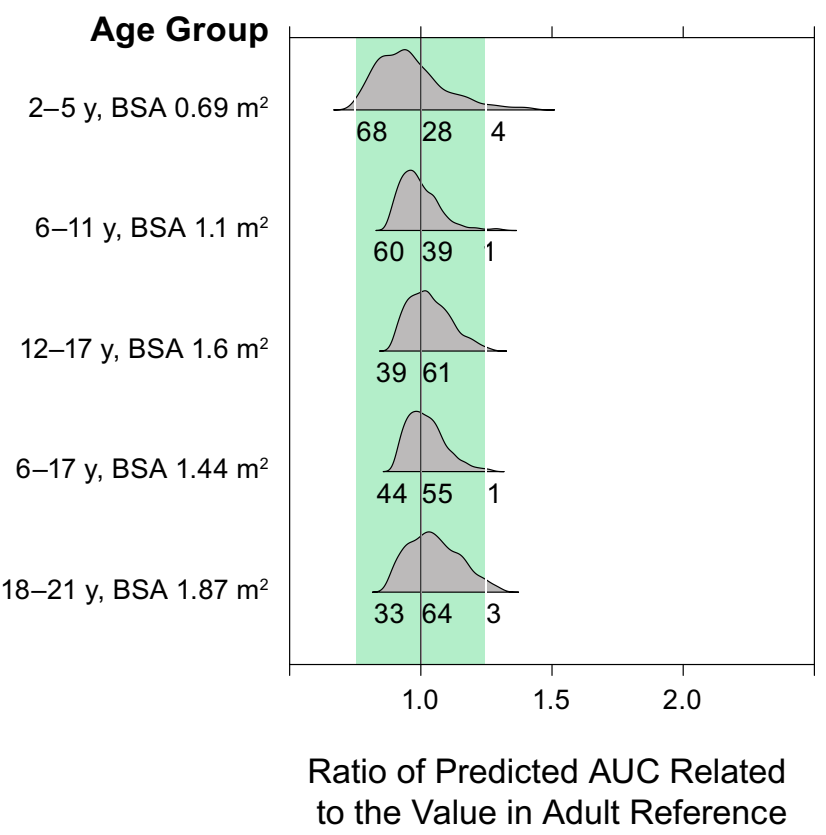

of the plots representing probability. The reference is the geometric mean steady-state $24 \mathrm{~h}$ sunitinib and SU012662 AUCs of 1233 and $551 \mathrm{ng} \bullet \mathrm{h} / \mathrm{ml}$, respectively, in an adult patient with GIST receiving a sunitinib dose of $50 \mathrm{mg}$. The green shaded areas represent the 0.75 1.25 ratio range. $A U C$ area under the curve, $B S A$ body surface area, GIST gastrointestinal stromal tumor

meaning lower sunitinib and SU012662 exposure. Population pharmacokinetic analysis using nonlinear mixed-effects modeling is an appropriate method for obtaining pharmacokinetic information in children, from both a practical and ethical standpoint [16]. Modeling and simulations can also be used for providing the rationale of drug dosing in specific populations, including children [17].

Based on the sunitinib and SU012662 apparent clearance in the final pharmacokinetic models, for patients aged 2-5 years with median $\mathrm{BSA}=0.69 \mathrm{~m}^{2}$, the predicted sunitinib doses expected to achieve steady-state total plasma exposures for sunitinib and SU012662 over the dosing interval similar to those in adults receiving $50 \mathrm{mg}$ on schedule $4 / 2$ are 25 and $22 \mathrm{mg} / \mathrm{m}^{2}$, respectively. For patients aged 6-11 years (median BSA $=1.1 \mathrm{~m}^{2}$ ), the predicted sunitinib doses are 22 and $21 \mathrm{mg} / \mathrm{m}^{2}$ with respect to sunitinib and SU012662 total plasma exposures, respectively. For patients aged 12-17 years (median BSA $=1.6 \mathrm{~m}^{2}$ ), the predicted sunitinib doses are $20 \mathrm{mg} / \mathrm{m}^{2}$ with respect to both sunitinib and SU012662 total plasma exposures. A dose of $\sim 20 \mathrm{mg} / \mathrm{m}^{2} /$ day in patients aged 6-17 years would therefore be expected to lead to similar total plasma exposures for sunitinib and SU012662 as for an adult receiving a dose of $50 \mathrm{mg} /$ day on schedule 4/2. 
As noted, to the best of our knowledge, this is the first population pharmacokinetic analysis of sunitinib in pediatric patients with GIST or solid tumors; therefore, comparison with other studies is limited. However, the dose of $20 \mathrm{mg} /$ $\mathrm{m}^{2}$ identified in the current study that would be expected to lead to similar sunitinib and SU012622 plasma exposures as a dose of $50 \mathrm{mg} / \mathrm{day}$ in adults on schedule $4 / 2$ is similar to the projected dose identified based on the extrapolation of pharmacokinetic and pharmacodynamic data for pediatric patients with GIST (data on file).

Due to slow enrolment of pediatric patients with GIST, only six patients completed the original study [15]. Therefore, the dataset in the current study with respect to pediatric patients with GIST comes from a smaller patient population. However, the population analysis approach used took advantage of pharmacokinetic data in other solid tumor settings to characterize further the pharmacokinetics of sunitinib and SU012662 in pediatric patients with GIST. In fact, as part of the covariate analysis, tumor type (GIST versus other solid tumor) was found to have no impact on the apparent clearance of sunitinib and SU012662 in pediatric patients. Although the lack of effect of tumor type on PK parameters may have occurred because of the small number of patients with GIST, it is notable that the central tendency of post-hoc estimates for $\mathrm{CL} / \mathrm{F}$ and $\mathrm{Vc} / \mathrm{F}$ between patients with GIST compared with those with other solid tumors were similar (CL/F: 25.3 versus 22.5 l/h; Vc/F: 993 versus 903 l, respectively), further indicating a lack of tumor effect in children. However, in an analysis of 647 adult patients comprising 395 patients with advanced RCC and 252 with GIST, there were higher $\mathrm{CL} / \mathrm{F}$ and $\mathrm{Vc} / \mathrm{F}$ values in patients with GIST compared with patients with advanced RCC [9]. The authors suggested that the differences were most likely due to the lower bioavailablity of sunitinib in the patients with GIST compared with those with advanced RCC. In turn, the lower bioavailability in adult patients with GIST could be caused by the fact that the site of disease in these patients potentially impacts the absorption of sunitinib from the gastrointestinal (GI) tract. Considering that different sites of the GI tract are affected in pediatric versus adult GIST, it is likely that the lack of tumor type effect (GIST versus other solid tumors) in pediatric patients in contrast to adult patients is due to the impact of disease site in the GI tract, affecting sunitinib absorption in adults but not pediatric patients.

In the dose escalation study in pediatric patients with solid tumors (study ADVL0612), the maximum tolerated sunitinib dose was determined to be $15 \mathrm{mg} / \mathrm{m}^{2}[12,13]$. In this study, the maximum tolerated dose projection was conducted in heavily pretreated pediatric patients, the majority of whom had tumors of the CNS. In the phase 2 study of patients with recurrent or refractory high-grade glioma or ependymoma (study ACNS1021), administration of sunitinib at a dose of $15 \mathrm{mg} / \mathrm{m}^{2}$ was generally well tolerated, with most adverse events of mild to moderate severity [14]. In study A6181196 in pediatric patients with GIST, although the starting dose was $15 \mathrm{mg} / \mathrm{m}^{2}$, it was escalated to $22.5 \mathrm{mg} / \mathrm{m}^{2}$ in five of six patients and escalated further to $30 \mathrm{mg} / \mathrm{m}^{2}$ in two out of these five patients [15]. The average dose across the study was close to $20 \mathrm{mg} / \mathrm{m}^{2}$ [6]. Therefore, based on the available data, and specifically safety data from study A6181196, a starting dose of 20 $\mathrm{mg} / \mathrm{m}^{2}$ is expected to be well tolerated in pediatric patients with GIST. Furthermore, according to BSA-tiered dosing in pediatric patients, doses of $12.5 \mathrm{mg}, 25.0 \mathrm{mg}, 37.5 \mathrm{mg}$, and $50.0 \mathrm{mg}$ in patients with BSA of $\leq 0.7 \mathrm{~m}^{2}, 0.8-1.5$ $\mathrm{m}^{2}, 1.6-2.4 \mathrm{~m}^{2}$, and $\geq 2.5 \mathrm{~m}^{2}$, respectively, are required to achieve the steady-state sunitinib (and SU012662) AUC approximately similar to that observed in adult patients with GIST receiving $50 \mathrm{mg} /$ day. It should also be noted that dosing by BSA is widely used in pediatric patients and that doses could be escalated if tolerability permits.

\section{Conclusion}

This population pharmacokinetic analysis of sunitinib in pediatric patients with GIST or solid tumors showed that BSA was the only covariate to statistically significantly affect sunitinib and SU012662 pharmacokinetic parameters. In a pediatric patient with GIST aged $6-17$ years, a sunitinib dose of $\sim 20 \mathrm{mg} / \mathrm{m}^{2} /$ day would be expected to lead to similar total plasma exposure of sunitinib and SU012662 compared with an adult patient with GIST receiving $50 \mathrm{mg} /$ day on schedule $4 / 2$. This dose needs to be viewed in context with tolerability data obtained from clinical trials of sunitinib in children [12-15]. The projected dose is supported by safety and tolerability data from pediatric patients with GIST in study A6181196 [15]. The results of this analysis help to bridge clinical pharmacology information between pediatric and adult patient populations with GIST and provide new information to guide dosing decisions for children with GIST.

Supplementary Information The online version contains supplementary material available at https://doi.org/10.1007/s13318-021-00671-7.

Acknowledgements Pfizer thank the Children's Oncology Group for conducting studies ADVL0612 and ACNS1021 and allowing Pfizer to use their data. Medical writing support was provided by David Cope, $\mathrm{PhD}$, of Engage Scientific Solutions and funded by Pfizer.

Author contributions Conception and design: EW, RK; development of methodology: EW, RK; acquisition of data: EW, SGD, CW, ACV, RK; analysis and interpretation of data: EW, RK; writing, review, and/or revision of the manuscript: $\mathrm{EW}, \mathrm{SGD}, \mathrm{CW}, \mathrm{ACV}, \mathrm{RK}$; clinical involvement in the original trials: SGD, CW, ACV. 


\section{Declarations}

Funding This study was sponsored by Pfizer.

Conflicts of Interest EW and RK are employees of Pfizer and hold stock or stock options with Pfizer. SGD has received travel expenses from Roche, Salarius, and Loxo Oncology, and consulting fees from Bayer and Loxo Oncology. CW has received research support and prior travel expenses from Eli Lilly \& Co, and is an employee Exelixis Inc and holds stock or stock options with Exelixis Inc. ACV is a consultant for Pfizer.

Ethical Approval The original clinical trials were conducted in accordance with the Declaration of Helsinki and all local regulatory requirements, and in compliance with Good Clinical Practice Guidelines from the International Conference on Harmonization.

Consent to Participate All patients or their parent or legal guardian provided written informed consent prior to participating in the original clinical trials.

\section{Consent to Publish Not applicable.}

Availability of Data and Material Upon request, and subject to certain criteria, conditions and exceptions (see https://www.pfizer.com/scien ce/clinical-trials/trial-data-and-results for more information), Pfizer will provide access to individual de-identified participant data from Pfizer-sponsored global interventional clinical studies conducted for medicines, vaccines and medical devices (1) for indications that have been approved in the US and/or EU or (2) in programs that have been terminated (i.e., development for all indications has been discontinued). Pfizer will also consider requests for the protocol, data dictionary and statistical analysis plan. Data may be requested from Pfizer trials 24 months after study completion. The de-identified participant data will be made available to researchers whose proposals meet the research criteria and other conditions, and for which an exception does not apply, via a secure portal. To gain access, data requestors must enter into a data access agreement with Pfizer.

Code Availability Not applicable.

Open Access This article is licensed under a Creative Commons Attribution-NonCommercial 4.0 International License, which permits any non-commercial use, sharing, adaptation, distribution and reproduction in any medium or format, as long as you give appropriate credit to the original author(s) and the source, provide a link to the Creative Commons licence, and indicate if changes were made. The images or other third party material in this article are included in the article's Creative Commons licence, unless indicated otherwise in a credit line to the material. If material is not included in the article's Creative Commons licence and your intended use is not permitted by statutory regulation or exceeds the permitted use, you will need to obtain permission directly from the copyright holder. To view a copy of this licence, visit http://creativecommons.org/licenses/by-nc/4.0/.

\section{References}

1. Mendel DB, Laird AD, Xin X, Louie SG, Christensen JG, Li $\mathrm{G}$, et al. In vivo antitumor activity of SU11248, a novel tyrosine kinase inhibitor targeting vascular endothelial growth factor and platelet-derived growth factor receptors: determination of a pharmacokinetic/pharmacodynamic relationship. Clin Cancer Res. 2003;9:327-37.
2. Chow LQ, Eckhardt SG. Sunitinib: from rational design to clinical efficacy. J Clin Oncol. 2007;25:884-96.

3. Faivre S, Demetri G, Sargent W, Raymond E. Molecular basis for sunitinib efficacy and future clinical development. Nat Rev Drug Discov. 2007;6:734-45.

4. Mena AC, Pulido EG, Guillen-Ponce C. Understanding the molecular-based mechanism of action of the tyrosine kinase inhibitor: sunitinib. Anticancer Drugs. 2010;21(suppl 1):S3-11.

5. Pfizer Inc. Sutent ${ }^{\circledR}$ (sunitinib malate) prescribing information. Pfizer, New York, NY. 2006. http://labeling.pfizer.com/showlabeli ng.aspx?id=607. Accessed 5 Nov 2019.

6. European Medicines Agency. Sutent ${ }^{\circledR}$ (sunitinib) summary of product characteristics. European Medicines Agency, Sandwich, UK. 2009. https://www.ema.europa.eu/documents/product-information/ sutent-epar-product-information_en.pdf. Accessed 5 Nov 2019.

7. Houk BE, Bello CL, Kang D, Amantea M. A population pharmacokinetic meta-analysis of sunitinib malate (SU11248) and its primary metabolite (SU12662) in healthy volunteers and oncology patients. Clin Cancer Res. 2009;15:2497-506.

8. Houk BE, Bello CL, Poland B, Rosen LS, Demetri GD, Motzer RJ. Relationship between exposure to sunitinib and efficacy and tolerability endpoints in patients with cancer: results of a pharmacokinetic/pharmacodynamic meta-analysis. Cancer Chemother Pharmacol. 2010;66:357-71.

9. Khosravan R, Motzer RJ, Fumagalli E, Rini BI. Population pharmacokinetic/pharmacodynamic modeling of sunitinib by dosing schedule in patients with advanced renal cell carcinoma or gastrointestinal stromal tumor. Clin Pharmacokinet. 2016;55:1251-69.

10. Lindauer A, Di Gion P, Kanefendt F, Tomalik-Scharte D, Kinzig M, Rodamer M, et al. Pharmacokinetic/pharmacodynamic modeling of biomarker response to sunitinib in healthy volunteers. Clin Pharmacol Ther. 2010;87:601-8.

11. Wang E, DuBois SG, Wetmore C, Khosravan R. Population pharmacokinetics-pharmacodynamics of sunitinib in pediatric patients with solid tumors. Cancer Chemother Pharmacol. 2020;86:181-92.

12. Dubois SG, Shusterman S, Ingle AM, Ahern CH, Reid JM, Wu $\mathrm{B}$, et al. Phase I and pharmacokinetic study of sunitinib in pediatric patients with refractory solid tumors: a Children's Oncology Group study. Clin Cancer Res. 2011;17:5113-22.

13. DuBois SG, Shusterman S, Reid JM, Ingle AM, Ahern $\mathrm{CH}$, Baruchel S, et al. Tolerability and pharmacokinetic profile of a sunitinib powder formulation in pediatric patients with refractory solid tumors: a Children's Oncology Group study. Cancer Chemother Pharmacol. 2012;69:1021-7.

14. Wetmore C, Daryani VM, Billups CA, Boyett JM, Leary S, Tanos R, et al. Phase II evaluation of sunitinib in the treatment of recurrent or refractory high-grade glioma or ependymoma in children: a Children's Oncology Group study ACNS1021. Cancer Med. 2016;5:1416-24.

15. Verschuur AC, Bajciova V, Mascarenhas L, Khosravan R, Lin X, Ingrosso A, et al. Sunitinib in pediatric patients with advanced gastrointestinal stromal tumor: results from a phase I/II trial. Cancer Chemother Pharmacol. 2019;84:41-50.

16. European Medicines Agency. Guideline on the role of pharmacokinetics in the development of medicinal products in the paediatric population. Committee For Medicinal Products For Human Use, London. 2007. https://www.ema.europa.eu/en/ documents/scientific-guideline/guideline-role-pharmacokinetics-development-medicinal-products-paediatric-population_en. pdf. Accessed 5 Nov 2019.

17. U.S. Food and Drug Administration. General clinical pharmacology consideration for pediatric studies for drugs and biological products. Guidcance for industry. U.S. Department of Health and Human Services, Center for Drug Evaluation and Research (CDER), Bethesda, MD. 2014. https://www.fda.gov/media/90358/ download. Accessed 5 Nov 2019. 